\title{
A certain $q$-Ruscheweyh type derivative operator and its applications involving multivalent functions
}

\author{
Bilal Khan ${ }^{1}$, H.M. Srivastava ${ }^{2,3,4,5}$, Sama Arjika ${ }^{6,7^{*}}$ (D), Shahid Khan ${ }^{8}$, Nazar Khan ${ }^{9}$ and Qazi Zahoor Ahmad ${ }^{10}$
}

\begin{tabular}{l}
\hline${ }^{\text {"Correspondence: }}$ \\
rjksama2008@gmail.com \\
${ }^{6}$ Department of Mathematics and \\
Informatics, University of Agadez, \\
Agadez, Niger \\
${ }^{7}$ International Chair of \\
Mathematical Physics and \\
Applications (ICMPA-UNESCO \\
Chair), University of Abomey-Calavi, \\
Post Box 072, Cotonou 50, Benin \\
Full list of author information is \\
available at the end of the article
\end{tabular}

\section{"Correspondence:}

${ }^{6}$ Department of Mathematics and Informatics, University of Agadez, Agadez, Niger

Chair), University of Abomey-Calavi, Full list of author information is available at the end of the article

\begin{abstract}
In the present paper, by using the concept of convolution and $q$-calculus, we define a certain $q$-derivative (or $q$-difference) operator for analytic and multivalent (or $p$-valent) functions. This presumably new $q$-derivative operator is an extension of the known $q$-analogue of the Ruscheweyh derivative operator. We also give some interesting applications of this $q$-derivative operator for multivalent functions by using the method of differential subordination. Relevant connections with a number of earlier works on this subject are also pointed out.
\end{abstract}

MSC: Primary 30C45; 30C50; 30C80; secondary 11B65; 47B38

Keywords: Analytic functions; Multivalent (or $p$-valent) functions; Hadamard product (or convolution); $q$-Derivative (or q-Difference) operator; $q$-Analogue of Ruscheweyh's derivative operator; Differential subordination

\section{Introduction, definitions, and motivation}

Let $\mathcal{A}(p)$ denote the class of multivalent (or $p$-valent) functions of the form:

$$
f(z)=z^{p}+\sum_{n=2}^{\infty} a_{n+p-1} z^{n+p-1} \quad(p \in \mathbb{N}=\{1,2,3, \ldots\})
$$

which are analytic in the open unit disk $\mathbb{U}$ given by

$$
\mathbb{U}=\{z: z \in \mathbb{C} \text { and }|z|<1\} .
$$

We note that

$$
\mathcal{A}(1)=: \mathcal{A} \text {. }
$$

(c) The Author(s) 2021. This article is licensed under a Creative Commons Attribution 4.0 International License, which permits use, sharing, adaptation, distribution and reproduction in any medium or format, as long as you give appropriate credit to the original author(s) and the source, provide a link to the Creative Commons licence, and indicate if changes were made. The images or other third party material in this article are included in the article's Creative Commons licence, unless indicated otherwise in a credit line to the material. If material is not included in the article's Creative Commons licence and your intended use is not permitted by statutory regulation or exceeds the permitted use, you will need to obtain permission directly from the copyright holder. To view a copy of this licence, visit http://creativecommons.org/licenses/by/4.0/. 
Definition 1 The Hadamard product or convolution of the following two functions $f_{j}(z) \in$ $\mathcal{A}(p)(j=1,2)$

$$
f_{j}(z)=z^{p}+\sum_{n=2}^{\infty} a_{n+p-1, j} z^{n+p-1} \quad(p \in \mathbb{N})
$$

is given by

$$
\left(f_{1} * f_{2}\right)(z)=z^{p}+\sum_{n=2}^{\infty} a_{n+p-1,1} a_{n+p-1,2} z^{n+p-1}
$$

Definition 2 For two analytic functions $f_{j}(j=1,2)$ in $\mathbb{U}$, the function $f_{1}$ is said to be subordinate to the function $f_{2}$, which is written as follows:

$$
f_{1} \prec f_{2} \quad \text { or } \quad f_{1}(z) \prec f_{2}(z) \quad(z \in \mathbb{U}) \text {, }
$$

if we can find a Schwartz function $w$, analytic in $\mathbb{U}$, with

$$
w(0)=0 \quad \text { and } \quad|w(z)|<1
$$

such that

$$
f_{1}(z)=f_{2}(w(z)) .
$$

Further, if the function $f_{2}$ is univalent in $\mathbb{U}$, then the following equivalence relation holds true:

$$
f_{1}(z) \prec f_{2}(z) \quad(z \in \mathbb{U}) \quad \Longleftrightarrow \quad f_{1}(0)=f_{2}(0) \quad \text { and } \quad f_{1}(\mathbb{U}) \subset f_{2}(\mathbb{U})
$$

We denote by $\mathcal{P}(\beta)$ the class of functions of the form

$$
\varphi(z)=1+c_{1} z+c_{2} z^{2}+\cdots,
$$

which are analytic in $\mathbb{U}$ and satisfy the following inequalities:

$$
\Re(\varphi(z))>\beta \quad(0 \leqq \beta<1) .
$$

It can be seen that

$$
\mathcal{P}(0)=\mathcal{P}
$$

where $\mathcal{P}$ is the well-known class of Carathéodory functions (see, for details, [6] and [49]). We next define the function $h(A, B ; z)$ given by (see [3])

$$
h(A, B ; z)=\frac{1+A z}{1+B z} \quad(z \in \mathbb{U} ;-1 \leqq B \leqq A \leqq 1) .
$$


This function $h(A, B ; z)$ is known to be the conformal map of the unit disk $\mathbb{U}$ to a circle which is symmetrical with respect to the real axis having the center at $\frac{1-A B}{1-B^{2}}(B \neq \pm 1)$ and the radius equal to $\frac{A-B}{1-B^{2}}(B \neq \pm 1)$.

In order to present some of the noteworthy and useful details of the definitions and principles of $q$-difference calculus, we assume throughout this article that

$$
0<q<1 \quad \text { and } \quad p \in \mathbb{N}=\{1,2,3, \ldots\} \text {. }
$$

Definition 3 For $f \in \mathcal{A}$, the $q$-difference (or the $q$-derivative) operator $\mathfrak{D}_{q}$ in a given subset of the set $\mathbb{C}$ of complex numbers is defined by (see [9] and [10])

$$
\left(\mathfrak{D}_{q} f\right)(z)= \begin{cases}\frac{f(z)-f(q z)}{(1-q) z} & (z \neq 0) \\ f^{\prime}(0) & (z=0)\end{cases}
$$

provided that $f^{\prime}(0)$ exists.

It is readily observed from equation (1.2) that

$$
\lim _{q \rightarrow 1-}\left(\mathfrak{D}_{q} f\right)(z)=\lim _{q \rightarrow 1-} \frac{f(z)-f(q z)}{(1-q) z}=f^{\prime}(z)
$$

for a differentiable function $f$ in a given subset of the complex space $\mathbb{C}$ (see also [37], [39] and [40] for some recent applications of the $q$-difference operators in the theory of $q$-series and $q$-polynomials).

Definition 4 Let $q \in(0,1)$ and define the $q$-number $[\tau]_{q}$ as follows:

$$
[\tau]_{q}= \begin{cases}\frac{1-q^{\tau}}{1-q} & (\tau \in \mathbb{C}) \\ \sum_{k=0}^{n-1} q^{k} & (\tau=n \in \mathbb{N}) \\ 0 & (\tau=0)\end{cases}
$$

It follows from Definition 4 with $\tau=n(n \in \mathbb{N})$ that

$$
[n]_{q} != \begin{cases}1 & (n=0), \\ \prod_{k=1}^{n}[k]_{q} & (n \in \mathbb{N}) .\end{cases}
$$

Definition 5 The generalized $q$-Pochhammer symbol given by

$$
[\tau]_{n, q} \quad\left(\tau \in \mathbb{C} ; n \in \mathbb{N}_{0}:=\mathbb{N} \cup\{0\} ;|q|<1\right)
$$

is defined as follows:

$$
[\tau]_{n, q}=\frac{\left(q^{\tau} ; q\right)_{n}}{(1-q)^{n}}= \begin{cases}1 & (n=0) \\ {[\tau]_{q}[\tau+1]_{q}[\tau+2]_{q} \cdots[\tau+n-1]_{q}} & (n \in \mathbb{N}) .\end{cases}
$$


Moreover, the $q$-gamma function $\Gamma_{q}(z)$ satisfies the following recurrence relation:

$$
\Gamma_{q}(z+1)=[z]_{q} \Gamma_{q}(z) \quad \text { and } \quad \Gamma_{q}(1)=1,
$$

where

$$
\Gamma_{q}(z)=(1-q)^{1-z} \prod_{n=0}^{\infty}\left(\frac{1-q^{n+1}}{1-q^{n+z}}\right) .
$$

The intensive applications of the $q$-calculus in exploring new directions in various diverse areas of mathematics and physics have fascinated a number of researchers to work in several distinctive areas of the mathematical and physical sciences. The $q$-derivative $\left(\mathfrak{D}_{q}\right)$ operator's versatile applications makes it significantly more important. Initially, in the year 1990, Ismail et al. [8] presented the idea of a $q$-extension of the class $\mathcal{S}^{*}$ of starlike functions. However, historically speaking, in the article [32] published in 1989, Srivastava gave a firm footing of the usages of the $q$-calculus and the basic (or $q$-) hypergeometric functions:

$$
{ }_{\mathfrak{v}} \Phi_{\mathfrak{s}}\left(\mathfrak{v}, \mathfrak{s} \in \mathbb{N}_{0}=\{0,1,2, \ldots\}\right)
$$

in the study of geometric function theory (GFT) (see, for details, [32]). More recently, the state-of-the-art survey and applications of the operators of the $q$-calculus and the fractional $q$-calculus, such as the $q$-derivative operator and the fractional $q$-derivative operators in geometric function theory of complex analysis, were investigated in a survey-cumexpository review article by Srivastava [33]. In this same survey-cum-expository review article, Srivastava [33] revealed and exposed the triviality of the so-called $(p, q)$-calculus associated with an obviously redundant and inconsequential additional parameter $p$ (see, for details, [33, p. 340]).

The aforementioned works [8] and [33] have inspired a number of researchers to contribute significantly in geometric function theory of complex analysis. Several convolution and fractional $q$-operators, which have been already defined, were surveyed in the above-cited work [33]. For example, Kanas and Răducanu [11] introduced the $q$-analogue of Ruscheweyh's derivative operator and, by using the concept of the Hadamard product (or convolution), Srivastava et al. [45] introduced a q-extension of Noor's integral operator and studied some of its applications. Aldweby [2] and Sokól [21] studied some classes of analytic functions defined by means of the $q$-analogue of Ruscheweyh's derivative operator. Many $q$-derivative and $q$-integral operators can be written in terms of the Hadamard product (or convolution). For details, we refer the reader to the earlier works $[1,7,18,21,23]$. Moreover, several authors (see, for example, [13, 19, 42, 47, 48]) have concentrated upon the classes of $q$-starlike functions related with the Janowski and other functions from several different viewpoints and the references cited therein. For some more recent investigations involving $q$-calculus, we may refer the interested reader to $[4,5,12,14-17,20,22,26-28,31,34-36,38,43,46]$.

In this paper, we first define an extended $q$-analogue of Ruscheweyh's derivative operator for multivalent (or $p$-valent) functions. In order to define this extended $q$-analogue of Ruscheweyh's derivative operator, we use the concepts of the Hadamard product (or 
convolution). We then give some interesting applications of this operator for multivalent functions by making use of the method of differential subordination. The extended $q$ analogue of Ruscheweyh's derivative operator is defined below.

Definition 6 For $f \in \mathcal{A}(p)$, the extended $q$-derivative operator $\mathcal{R}_{q}^{\lambda+p-1}: \mathcal{A}(p) \rightarrow \mathcal{A}(p)$ for multivalent functions is defined as follows:

$$
\begin{aligned}
\mathcal{R}_{q}^{\lambda+p-1} f(z) & =Q_{\lambda, q}^{p}(z) * f(z) \\
& =z^{p}+\sum_{n=2}^{\infty} \frac{[\lambda+p]_{n-p, q}}{[n-p]_{q} !} a_{n+p-1} z^{n+p-1} \quad(\lambda>-1),
\end{aligned}
$$

where

$$
Q_{\lambda, q}^{p}(z)=z^{p-1}\left(z+\sum_{n=2}^{\infty} \frac{[\lambda+p]_{n-p, q}}{[n-p]_{q} !} z^{n}\right) .
$$

The following identity can easily be verified by using (1.3):

$$
q^{\lambda} z \mathfrak{D}_{q} \mathcal{R}_{q}^{\lambda+p-1} f(z)=[\lambda+p]_{q} \mathcal{R}_{q}^{\lambda+p} f(z)-[\lambda]_{q} \mathcal{R}_{q}^{\lambda+p-1} f(z) .
$$

It can also be seen that, by putting $p=1$ in (1.4), we have

$$
q^{\lambda} z \mathfrak{D}_{q} \mathcal{R}_{q}^{\lambda} f(z)=[\lambda+1]_{q} \mathcal{R}_{q}^{\lambda+1} f(z)-[\lambda]_{q} \mathcal{R}_{q}^{\lambda} f(z),
$$

which is the well-known relation studied by Kanas [11].

Remark 1 It is easily seen that, upon setting $p=1$, the extended $q$-analogue of Ruscheweyh's derivative operator $\mathcal{R}_{q}^{\lambda+p-1} f(z)$ reduces to the $q$-Ruscheweyh derivative operator which was studied by Kanas [11]. For $p=1$ and $q \rightarrow 1-$, the extended $q$-analogue of Ruscheweyh's derivative operator $\mathcal{R}_{q}^{\lambda+p-1} f(z)$ reduces to the familiar derivative operator introduced by Ruscheweyh [30]. Moreover, if we put $p=1$ and $\lambda=0$, we have

$$
\mathcal{R}_{q}^{0+1-1} f(z)=\mathcal{R}_{q}^{0} f(z)=f(z)
$$

\section{A set of lemmas}

To prove our main results, we need the following lemmas.

Lemma 1 (see [25]) Let $\varphi_{j} \in \mathcal{P}\left(\beta_{j}\right)$ be given by $(1.1)$ for $\left(0 \leqq \beta_{j}<1 ; j=1,2\right)$. Then

$$
\varphi_{1} * \varphi_{2} \in \mathcal{P}\left(\beta_{3}\right)
$$

where

$$
\beta_{3}=1-\left(1-\beta_{1}\right)\left(1-\beta_{2}\right) .
$$


Lemma 2 (see [24]) Let the function $\varphi$, given by (1.4), be in the class $\mathcal{P}(\beta)$. Then

$$
\Re(\varphi(z))>2 \beta-1+\frac{2(1-\beta)}{1+|z|} \quad(0 \leqq \beta<1) .
$$

Lemma 3 (see [29]) The function given by

$$
(1-z)^{\gamma}=e^{\gamma \log (1-z)} \quad(\gamma \neq 0)
$$

is univalent in $\mathbb{U}$ if and only if $\gamma$ is either in the closed disk $|\gamma-1| \leqq 1$ or in the closed disk $|\gamma+1| \leqq 1$

Lemma 4 (see [3]) Let the function $h(z)$ be analytic and convex univalent in $\mathbb{U}$ with $h(0)=$ 1. Also, let the function $g(z)$ given by

$$
g(z)=1+b_{1} z+b_{2} z^{2}+\cdots
$$

be analytic in $\mathbb{U}$. If

$$
g(z)+\frac{z \mathfrak{D}_{q} g(z)}{c} \prec h(z) \quad(z \in \mathbb{U} ; c \neq 0),
$$

then, for $\Re(c) \geqq 0$, the following subordination relation holds true:

$$
g(z) \prec \frac{c}{z^{c}} \int_{0}^{z} t^{c-1} h(t) d t .
$$

Lemma 5 (see [3]) Let the function $u(z)$ be univalent in $\mathbb{U}$, and let the functions $\theta(w)$ and $\varphi(w)$ be analytic in the domain $\mathbb{D}$ containing $u(\mathbb{U})$ with $\varphi(w) \neq 0$ when $w \in u(\mathbb{U})$. Set

$$
Q(z)=z \mathfrak{D}_{q}(u(z)) \varphi(u(z)) \quad \text { and } \quad h(z)=\theta(u(z)+Q(z))
$$

and suppose that

(i) $Q(z)$ is starlike univalent in $\mathbb{U}$.

(ii) $\mathfrak{\Re (}\left(\frac{z \mathfrak{D}_{q} h(z)}{Q(z)}\right)=\Re\left(\frac{z \mathfrak{D}_{q} \theta(u(z))}{(\varphi(u(z))}\right)+\left(\frac{z \mathfrak{D}_{q} Q(z)}{Q(z)}\right)>0$.

If $m(z)$ is analytic in $\mathbb{U}$,

$$
m(z)=u(0) \quad \text { and } \quad m(\mathbb{U}) \subset \mathbb{D}
$$

and

$$
\theta(m(z))+z \mathfrak{D}_{q}(m(z)) \varphi(m(z)) \prec \theta(u(z))+z \mathfrak{D}_{q}(u(z)) \varphi(u(z))=h(z),
$$

then

$$
m(z) \prec u(z) \quad(z \in \mathbb{U})
$$

and $u(z)$ is the best dominant. 


\section{Main results}

Our first main result in this section is stated as Theorem 1.

Theorem 1 Let $\lambda>0, \alpha>0$, and $-1 \leqq B \leqq A<1$. Iff $\in \mathcal{A}(p)$ satisfies the following subordination relation:

$$
(1-\alpha) \frac{\mathcal{R}_{q}^{\lambda+p-1} f(z)}{z^{p}}+\alpha \frac{\mathcal{R}_{q}^{\lambda+p} f(z)}{z^{p}} \prec h(A, B, z),
$$

then

$$
\begin{aligned}
& \mathfrak{R}\left(\left\{\frac{\mathcal{R}_{q}^{\lambda+p-1} f(z)}{z^{p}}\right\}^{\frac{1}{n}}\right) \\
& \quad \geqq\left(\frac{[\lambda+p]_{q}}{\alpha[p]_{q} q^{\lambda}} \int_{0}^{1} u^{\left(\frac{[\lambda+p]_{q}}{\alpha[p] q q^{\lambda}}\right)-1}\left(\frac{1-A u}{1-B u}\right) d u\right)^{\frac{1}{n}} \quad(n \geqq 1) .
\end{aligned}
$$

The result is sharp.

Proof Let

$$
g(z)=\frac{\mathcal{R}_{q}^{\lambda+p-1} f(z)}{z^{p}}
$$

Then, for $f \in \mathcal{A}(p)$, the function $g$ given by

$$
g(z)=1+b_{1} z+b_{2} z^{2}+\cdots \quad(z \in \mathbb{U})
$$

is analytic in $\mathbb{U}$. By using the logarithmic q-differentiation on both sides of (3.2) and multiplying the resulting equation by $z$, we have

$$
\frac{z \mathfrak{D}_{q}(g(z))}{g(z)}=\frac{z \mathcal{R}_{q}^{\lambda+p-1} f(z)}{\mathcal{R}_{q}^{\lambda+p-1} f(z)}-[p]_{q} .
$$

By making use of (1.4), we obtain

$$
\frac{z \mathfrak{D}_{q}(g(z))}{g(z)}=\frac{[\lambda+p]_{q}}{q^{\lambda}} \frac{\mathcal{R}_{q}^{\lambda+p} f(z)}{\mathcal{R}_{q}^{\lambda+p-1} f(z)}-\frac{[\lambda]_{q}}{q^{\lambda}}-[p]_{q} .
$$

Taking into account that

$$
[\lambda+p]_{q}=[\lambda]_{q}+[p]_{q} q^{\lambda}
$$

we get

$$
\frac{q^{\lambda}}{[\lambda+p]_{q}} z \mathfrak{D}_{q}(g(z))+g(z)=\frac{\mathcal{R}_{q}^{\lambda+p} f(z)}{z^{p}} .
$$

Also, from (1.4), (3.2), and (3.3), we have

$$
g(z)+\frac{\alpha q^{\lambda}[p]_{q}}{[\lambda+p]_{q}} z \mathfrak{D}_{q} g(z) \prec h(A, B, z) .
$$


Now, by applying Lemma 4, we find that

$$
g(z) \prec \frac{[\lambda+p]_{q}}{\alpha q^{\lambda}[p]_{q}} z^{-\frac{[\lambda+p] q}{\alpha[p] q q^{\lambda}}} \int_{0}^{1} u^{\left(\frac{[\lambda+p]_{q}}{\alpha[p] q q^{\lambda}}\right)-1}\left(\frac{1+A t}{1+B t}\right) d t .
$$

Also, by making use of the concept of subordination on (3.5), we have

$$
\left(\frac{\mathcal{R}_{q}^{\lambda+p-1} f(z)}{z^{p}}\right)=\frac{[\lambda+p]_{q}}{\alpha q^{\lambda}[p]_{q}} \int_{0}^{1} u^{\left(\frac{[\lambda+p]_{q}}{\alpha[p] q q^{\lambda}}\right)-1}\left(\frac{1+A u w(z)}{1+B u w(z)}\right) d u
$$

It follows from (3.6), together with $-1 \leqq B<A \leqq 1$ and $\lambda>0$, that

$$
\Re\left(\frac{\mathcal{R}_{q}^{\lambda+p-1} f(z)}{z^{p}}\right)>\frac{[\lambda+p]_{q}}{\alpha q^{\lambda}[p]_{q}} \int_{0}^{1} u^{\left(\frac{[\lambda+p]_{q}}{\alpha[p] q q^{\lambda}}\right)-1}\left(\frac{1-A u}{1-B u}\right) d u .
$$

Since

$$
\Re\left(w^{\frac{1}{n}}\right) \geqq\{\Re(w)\}^{1 / n} \quad(\Re(w)>0 ; n \geqq 1),
$$

by making use of concept (3.8), the inequality in (3.1) follows directly from (3.7).

In order to show the sharpness of (3.1), we define the function $f \in \mathcal{A}(p)$ by

$$
\frac{\mathcal{R}_{q}^{\lambda+p-1} f(z)}{z^{p}}=\frac{[\lambda+p]_{q}}{\alpha q^{\lambda}[p]_{q}} \int_{0}^{1} u^{\left(\frac{[\lambda+p] q}{\alpha[p]_{q} q^{\lambda}}\right)-1}\left(\frac{1+A u z}{1+B u z}\right) d u .
$$

For this function $f$ involved in (3.9), we find that

$$
(1-\alpha) \frac{\mathcal{R}_{q}^{\lambda+p-1} f(z)}{z^{p}}+\alpha \frac{\mathcal{R}_{q}^{\lambda+p} f(z)}{z^{p}}=\frac{1+A z}{1+B z}
$$

and

$$
\frac{\mathcal{R}_{q}^{\lambda+p-1} f(z)}{z^{p}} \rightarrow \frac{[\lambda+p]_{q}}{\alpha q^{\lambda}[p]_{q}} \int_{0}^{1} u^{\left(\frac{[\lambda+p]_{q}}{\alpha[p]_{q} q^{\lambda}}\right)-1}\left(\frac{1-A u}{1-B u}\right) d u \quad(z \rightarrow-1) .
$$

This completes the proof of Theorem 1.

Remark 2 If we set $p=1$ in Theorem 1 , we are led to the results similar to those given by Aldweby and Darus [3].

We next state and prove Theorem 2.

Theorem 2 Let $A=1-2 \alpha, B=-1, \alpha, \lambda>1, n \geqq 1$, and $0 \leqq \beta<1$. If the function $f \in \mathcal{A}(p)$ satisfies the following subordination condition:

$$
(1-\alpha) \frac{\mathcal{R}_{q}^{\lambda+p-1} f(z)}{z^{p}}+\alpha \frac{\mathcal{R}_{q}^{\lambda+p} f(z)}{z^{p}} \prec h(1-2 \alpha,-1, z),
$$


then

$$
\begin{aligned}
& \Re\left(\left\{\frac{\mathcal{R}_{q}^{\lambda+p-1} f(z)}{z^{p}}\right\}^{\frac{1}{n}}\right) \\
& \quad>\left((2 \beta-1) u^{\frac{[\lambda+p]_{q}}{\alpha[p] q q^{\lambda}}}+\frac{2(1-\beta)[\lambda+p]_{q}}{\alpha[p]_{q} q^{\lambda}} \int_{0}^{1} \frac{u^{\left(\frac{[\lambda+p] q}{\alpha[p] q q^{\lambda}}\right)-1}}{1+u} d u\right)^{\frac{1}{n}} .
\end{aligned}
$$

Proof Following the same steps as those in the proof of Theorem 1 and considering the function $g$ given by

$$
g(z)=\frac{\mathcal{R}_{q}^{\lambda+p-1} f(z)}{z^{p}},
$$

the differential subordination in (3.4) becomes

$$
g(z)+\frac{\alpha q^{\lambda}[p]_{q}}{[\lambda+p]_{q}} z \mathfrak{D}_{q} g(z) \prec \frac{1+(2 \beta-1) z}{1+z} .
$$

Therefore, we have

$$
\begin{aligned}
\Re & \left(\left\{\frac{\mathcal{R}_{q}^{\lambda+p-1} f(z)}{z^{p}}\right\}^{\frac{1}{n}}\right) \\
& >\left(\frac{[\lambda+p]_{q}}{\alpha q^{\lambda}[p]_{q}} \int_{0}^{1} u^{\left(\frac{[\lambda+p]_{q}}{\alpha[p] q q^{\lambda}}\right)-1}\left(\frac{1+(2 \beta-1) u}{1+u}\right) d u\right)^{\frac{1}{n}} \\
& =\left(\frac{[\lambda+p]_{q}}{\alpha q^{\lambda}[p]_{q}} \int_{0}^{1} u^{\left(\frac{[\lambda+p] q}{\alpha[p] q q^{\lambda}}\right)-1}\left((2 \beta-1)+\frac{2(1-\beta) u}{1+u}\right) d u\right)^{\frac{1}{n}} \\
& =\left((2 \beta-1) u^{\left(\frac{[\lambda+p]_{q}}{\alpha[p] q q^{\lambda}}\right)-1}+\frac{2(1-\beta)[\lambda+p]_{q}}{\alpha q^{\lambda}[p]_{q}} \int_{0}^{1} \frac{u^{\left(\frac{\lambda \lambda+p]_{q}}{\alpha[p]_{q} q^{\lambda}}\right)-1}}{1+u} d u\right)^{\frac{1}{n}} .
\end{aligned}
$$

Our demonstration of Theorem 2 is now completed.

Theorem 3 Let $\lambda>0$ and $0 \leqq \rho<1$. Also, let the parameter $\gamma \in \mathbb{C} \backslash\{0\}$ satisfy either

$$
\left|2 \gamma(1-\rho)\left(\frac{[\lambda+p]_{q}}{\alpha q^{\lambda}[p]_{q}}-1\right)\right| \leqq 1
$$

or

$$
\left|2 \gamma(1-\rho)\left(\frac{[\lambda+p]_{q}}{\alpha q^{\lambda}[p]_{q}}+1\right)\right| \leqq 1 .
$$

If $\in \mathcal{A}(p)$ satisfies the following inequality:

$$
\Re\left(\frac{\mathcal{R}_{q}^{\lambda+p} f(z)}{\mathcal{R}_{q}^{\lambda+p-1} f(z)}\right)>\rho,
$$


then

$$
\Re\left(\frac{\mathcal{R}_{q}^{\lambda+p-1} f(z)}{z^{p}}\right)^{\gamma} \prec \frac{1}{(1-z)^{2 \gamma(1-\rho) \frac{[\lambda+p]_{q}}{\alpha q^{\lambda}[p] q}}},
$$

where $u(z)$ is the best dominant.

Proof Let

$$
m(z)=\left(\frac{\mathcal{R}_{q}^{\lambda+p-1} f(z)}{z^{p}}\right)^{\gamma}
$$

Then, by making use of (1.4), (3.10), and (3.11), we obtain

$$
1+\frac{q^{\lambda}[p]_{q}}{\gamma[\lambda+p]_{q}} \frac{z \mathfrak{D}_{q}(g(z)}{g(z)} \prec \frac{1+(1-2 \rho) z}{1-z} .
$$

We now assume that

$$
u(z)=\frac{1}{(1-z)^{2 \gamma(1-\rho) \frac{[\lambda+p]_{q}}{q^{\lambda}[p]_{q}}}}, \quad \theta(w)=1 \quad \text { and } \quad \varphi(w)=\frac{q^{\lambda}[p]_{q}}{\gamma[\lambda+p]_{q} w} .
$$

Then, by Lemma $3, u(z)$ is univalent in $\mathbb{U}$. Further, it is easy to show that the functions $u(z)$, $\theta(w)$, and $\varphi(w)$ satisfy the condition of Lemma 5 . We note also that the functions given by

$$
Q(z)=z \mathfrak{D}_{q}(u(z)) \varphi(u(z))=\frac{2(1-\rho) z}{1-z}
$$

and

$$
h(z)=\theta(u(z)+Q(z))=\frac{1+(1-2 \rho) z}{1-z}
$$

are univalent starlike in $\mathbb{U}$. Hence, by finally combining (3.12) and Lemma 5 , we get the assertion of Theorem 3.

Remark 3 If we set $p=1$ in Theorem 3, we are led to the results similar to those given by Aldweby and Darus [3].

Theorem 4 Let $\lambda>0, \alpha<1$, and $-1 \leqq B_{j} \leqq A_{j}<1(j=1,2)$. If each of the functions $f_{j} \in \mathcal{A}(p)$ $(j=1,2)$ satisfies the following subordination condition:

$$
(1-\alpha) \frac{\mathcal{R}_{q}^{\lambda+p-1} f_{j}(z)}{z^{p}}+\alpha \frac{\mathcal{R}_{q}^{\lambda+p} f_{j}(z)}{z^{p}} \prec h\left(A_{j}, B_{j}, z\right) \quad(j=1,2),
$$

then

$$
(1-\alpha) \frac{\mathcal{R}_{q}^{\lambda+p-1} \Theta(z)}{z^{p}}+\alpha \frac{\mathcal{R}_{q}^{\lambda+p} \Theta(z)}{z^{p}} \prec h(1-2 \gamma,-1, z),
$$


where

$$
\Theta(z)=\mathcal{R}_{q}^{\lambda+p-1}\left(f_{1} * f_{2}\right)(z)
$$

and

$$
\gamma=1-\frac{4\left(A_{1}-B_{1}\right)\left(A_{2}-B_{2}\right)}{\left(1-B_{1}\right)\left(1-B_{2}\right)} \cdot\left(1-\frac{[\lambda+p]_{q}}{\alpha[p]_{q} q^{\lambda}} \int_{0}^{1} \frac{u^{\left(\frac{[\lambda+p]_{q}}{q^{\lambda}[p]_{q}}\right)-1}}{1+u} d u\right) .
$$

Proof We define the functions $h_{j}(j=1,2)$ by

$$
h_{j}=(1-\alpha) \frac{\mathcal{R}_{q}^{\lambda+p-1} f_{j}(z)}{z^{p}}+\alpha \frac{\mathcal{R}_{q}^{\lambda+p} f_{j}(z)}{z^{p}}
$$

where $f_{j} \in \mathcal{A}(p)(j=1,2)$. We have $h_{j} \in \mathcal{P}\left(\beta_{j}\right)(j=1,2)$, where

$$
\beta_{j}=\frac{1-A_{j}}{1-B_{j}} \quad(j=1,2)
$$

By making use of (1.4) and (3.14), we obtain

$$
\mathcal{R}_{q}^{\lambda+p-1} f_{j}(z)=\frac{[\lambda+p]_{q}}{\alpha q^{\lambda}[p]_{q}} z^{1-\left(\frac{[\lambda+p] q}{q^{\lambda}[p] q}\right)} \int_{0}^{1} t^{\left(\frac{[\lambda+p] q}{q^{\lambda}[p]_{q}}\right)-1} h_{j}(t) d t \quad(j=1,2)
$$

which, in light of (3.13), shows that

$$
\mathcal{R}_{q}^{\lambda+p-1} \Theta(z)=\frac{[\lambda+p]_{q}}{\alpha q^{\lambda}[p]_{q}} z^{1-\left(\frac{[\lambda+p]_{q}}{q^{\lambda}[p] q}\right)} \int_{0}^{1} t^{\left(\frac{[\lambda+p]_{q}}{q^{\lambda}[p]_{q}}\right)-1} h_{0}(t) d t
$$

where, for convenience,

$$
\begin{aligned}
h_{0} & =(1-\alpha) \frac{\mathcal{R}_{q}^{\lambda+p-1} \Theta(z)}{z^{p}}+\alpha \frac{\mathcal{R}_{q}^{\lambda+p} \Theta(z)}{z^{p}} \\
& =\frac{[\lambda+p]_{q}}{\alpha q^{\lambda}[p]_{q}} z^{1-\left(\frac{[\lambda+p]_{q}}{q^{\lambda}[p] q}\right)} \int_{0}^{1} t^{\left(\frac{[\lambda+p] q}{q^{\lambda}[p]_{q}}\right) 1}\left(h_{1} * h_{2}\right)(t) d t .
\end{aligned}
$$

If we apply Lemma 1 , we get $\left(h_{1} * h_{2}\right) \in \mathcal{P}\left(\beta_{3}\right)$, where

$$
\beta_{3}=1-2\left(1-\beta_{1}\right)\left(1-\beta_{2}\right)
$$

Now, with an application of Lemma 2, we have

$$
\begin{aligned}
\Re\left(h_{0}(z)\right) & =\frac{[\lambda+p]_{q}}{\alpha q^{\lambda}[p]_{q}} \int_{0}^{1} u^{\left(\frac{[\lambda+p]_{q}}{q^{\lambda}[p]_{q}}\right)-1} \Re\left(h_{1} * h_{2}\right)(u z) d u \\
& \geqq \frac{[\lambda+p]_{q}}{\alpha q^{\lambda}[p]_{q}} \int_{0}^{1} u^{\left(\frac{[\lambda+p]_{q}}{q^{\lambda}[p]_{q}}\right)-1}\left(\left(2 \beta_{3}-1\right)+\frac{2\left(1-\beta_{3}\right)}{1+u|z|}\right) d u
\end{aligned}
$$




$$
\begin{aligned}
& >\frac{[\lambda+p]_{q}}{\alpha q^{\lambda}[p]_{q}} \int_{0}^{1} u^{\left(\frac{[\lambda+p]_{q}}{q^{\lambda}[p] q}\right)-1}\left(\left(2 \beta_{3}-1\right)+\frac{2\left(1-\beta_{3}\right)}{1+u}\right) d u \\
& =1-\frac{4\left(A_{1}-B_{1}\right)\left(A_{2}-B_{2}\right)}{\left(1-B_{1}\right)\left(1-B_{2}\right)}\left(1-\frac{[\lambda+p]_{q}}{\alpha[p]_{q} q^{\lambda}} \int_{0}^{1} \frac{u^{\left(\frac{(\lambda+p]_{q}}{q^{\lambda}[p] q}\right)-1}}{1+u} d u\right) \\
& =\gamma,
\end{aligned}
$$

which leads us to the desired assertion of Theorem 4 .

Remark 4 If we set $p=1$ in Theorem 4 , we are led to the results similar to those given by Aldweby and Darus [3].

\section{Conclusion}

In our present work, we are motivated by the well-established usage of the basic (or $q$-) calculus and fractional basic (or $q$-) calculus in geometric function theory of complex analysis as described by Srivastava's survey-cum-expository review article [33]. The extended $q$ version of the $q$-Ruscheweyh type derivative operator for $p$-valent functions in $\mathbb{U}$ has been introduced here. We have also derived several interesting results for this newly defined $q$-operator. The importance of the results demonstrated in this paper lies in the demonstrated fact that these results would generalize and extend various previously known results derived in many earlier works.

Basic (or $q$-) polynomials and basic (or $q$-) series, especially the basic (or $q$-) hypergeometric functions and basic (or $q$-) hypergeometric polynomials, are relevant specially in many areas (see, for example, [41, pp. 350-351]; see also [28, 44], and [16]). Moreover, as we remarked above and in the introductory Sect. 1, based upon the recently-published survey-cum-expository review article by Srivastava [33], the so-called $(p, q)$-calculus is a relatively insignificant and inconsequential translation of the traditional $q$-calculus, the extra parameter $p$ being redundant or superfluous (see, for details, [33, p. 340]). This observation by Srivastava [33] will indeed apply also to any attempt to produce the rather straightforward $(p, q)$-variations of the results which we have presented in this paper.

Acknowledgements

Not applicable.

Availability of data and materials

Not applicable.

Competing interests

The authors declare that they have no competing interests.

Authors' contributions

All authors contributed equally to this manuscript and approved its final version.

\section{Author details}

'School of Mathematical Sciences and Shanghai Key Laboratory of PMMP, East China Normal University, 500 Dongchuan Road, Shanghai 200241, People's Republic of China. ${ }^{2}$ Department of Mathematics and Statistics, University of Victoria, Victoria, British Columbia V8W 3R4, Canada. ${ }^{3}$ Department of Medical Research, China Medical University Hospital, China Medical University, Taichung, 40402, Taiwan, Republic of China. ${ }^{4}$ Department of Mathematics and Informatics, Azerbaijan University, 71 Jeyhun Hajibeyli Street, AZ1007 Baku, Azerbaijan. ${ }^{5}$ Section of Mathematics, International Telematic University Uninettuno, I-00186 Rome, Italy. ${ }^{6}$ Department of Mathematics and Informatics, University of Agadez, Agadez, Niger. ${ }^{7}$ International Chair of Mathematical Physics and Applications (ICMPA-UNESCO Chair), University of

Abomey-Calavi, Post Box 072, Cotonou 50, Benin. ${ }^{8}$ Department of Mathematics, Riphah International University, Riphah, Islamabad, 747424, Pakistan. ${ }^{9}$ Department of Mathematics, Abbottabad University of Science and Technology, Abbottabad 22010, Pakistan. ${ }^{10}$ Government Akhtar Nawaz Khan (Shaheed) Degree College KTS, Haripur 22620, Pakistan. 


\section{Publisher's Note}

Springer Nature remains neutral with regard to jurisdictional claims in published maps and institutional affiliations.

\section{Received: 17 February 2021 Accepted: 28 May 2021 Published online: 06 June 2021}

\section{References}

1. Aldweby, H., Darus, M.: A subclass of harmonic univalent functions associated with q-analogue of Dziok-Srivastava operatror. ISRN Math. Anal. 2013, Article ID 382312 (2013)

2. Aldweby, H., Darus, M.: On harmonic meromorphic functions associated with basic hypergeometric functions. Sci. World J. 2013, Article ID 164287 (2013)

3. Aldweby, H., Darus, M.: Some subordination results on q-analogue of Ruscheweyh differential operator. Abstr. Appl. Anal. 2014, Article ID 958563 (2014)

4. Arif, M., Ahmad, K., Liu, J.-L.: Convolution properties for a family of analytic functions involving $q$-analogue of Ruscheweyh differential operator. Turk. J. Math. 43, 1712-1720 (2019)

5. Arif, M., Srivastava, H.M., Umar, S.: Some applications of a $q$-analogue of the Ruscheweyh type operator for multivalent functions. Rev. R. Acad. Cienc. Exactas Fís. Nat., Ser. A Mat. 113, 1211-1221 (2019)

6. Cho, N.E., Srivastava, H.M., Adegani, E.A., Motamednezhad, A.: Criteria for a certain class of the Carathéodory functions and their applications. J. Inequal. Appl. 2020, Article ID 85 (2020)

7. Hussain, S., Khan, S., Zaighum, M.A., Darus, M.: Applications of a q-Sălăgean type operator on multivalent function. J. Inequal. Appl. 2018, Article ID 301 (2018)

8. Ismail, M.E.-H., Merkes, E., Styer, D.: A generalization of starlike functions. Complex Var. Theory Appl. 14, 77-84 (1990)

9. Jackson, F.H.: On q-definite integrals. Q. J. Pure Appl. Math. 41, 193-203 (1910)

10. Jackson, F.H.: q-Difference equations. Am. J. Math. 32, 305-314 (1910)

11. Kanas, S., Răducanu, D.: Some class of analytic functions related to conic domains. Math. Slovaca 64, 1183-1196 (2014)

12. Khan, B., Liu, Z.-G., Srivastava, H.M., Khan, N., Darus, M., Tahir, M.: A study of some families of multivalent $q$-starlike functions involving higher-order q-derivatives. Mathematics 8, Article ID 1470 (2020)

13. Khan, B., Srivastava, H.M., Khan, N., Darus, M., Ahmad, Q.Z., Tahir, M.: Applications of certain conic domains to a subclass of $q$-starlike functions associated with the Janowski functions. Symmetry 13, Article ID 574 (2021)

14. Khan, B., Srivastava, H.M., Khan, N., Darus, M., Tahir, M., Ahmad, Q.Z.: Coefficient estimates for a subclass of analytic functions associated with a certain leaf-like domain. Mathematics 8, Article ID 1334 (2020)

15. Khan, B., Srivastava, H.M., Tahir, M., Darus, M., Ahmad, Q.Z., Khan, N.: Applications of a certain integral operator to the subclasses of analytic and bi-univalent functions. AlMS Math. 6, 1024-1039 (2021)

16. Khan, N., Shafiq, M., Darus, M., Khan, B., Ahmad, Q.Z.: Upper bound of the third Hankel determinant for a subclass of q-starlike functions associated with lemniscate of Bernoulli. J. Math. Inequal. 14, 51-63 (2020)

17. Khan, Q., Arif, M., Raza, M., Srivastava, G., Tang, H., Rehman, S.U., Ahmad, B.: Some applications of a new integral operator in q-analog for multivalent functions. Mathematics 7, Article ID 1178 (2019)

18. Khan, S., Hussain, S., Zaighum, M.A., Khan, M.M.: Some subclasses of analytic function in conical domain associated with Ruscheweyh q-differential operator. Int. J. Anal. Appl. 16, 239-253 (2018)

19. Mahmood, S., Ahmad, Q.Z., Srivastava, H.M., Khan, N., Khan, B., Tahir, M.: A certain subclass of meromorphically q-starlike functions associated with the Janowski functions. J. Inequal. Appl. 2019, Article ID 88 (2019)

20. Mahmood, S., Raza, N., Abujarad, E.S.A., Srivastava, G., Srivastava, H.M., Malik, S.N.: Geometric properties of certain classes of analytic functions associated with a q-integral operator. Symmetry 11, Article ID 719 (2019)

21. Mahmood, S., Sokól, J.: New subclass of analytic functions in conical domain associated with Ruscheweyh q-differential operator. Results Math. 71, 1-13 (2017)

22. Mahmood, S., Srivastava, H.M., Khan, N., Ahmad, Q.Z., Khan, B., Ali, I.: Upper bound of the third Hankel determinant for a subclass of $q$-starlike functions. Symmetry 11, Article ID 347 (2019)

23. Mohammed, A., Darus, M.: A generalized operator involving the $q$-hypergeometric function. Mat. Vesn. $65,454-465$ (2013)

24. Pashkouleva, D.Ž:: The starlikeness and spiral-convexity of certain subclasses of analytic functions. In: Srivastava, H.M., Owa, S. (eds.) Current Topics in Analytic Function Theory, pp. 266-273. World Scientific, Singapore (1992)

25. Ponnusamy, S., Singh, V:: Convolution properties of some classes of analytic functions. J. Math. Sci. 89, 1008-1020 (1998)

26. Raza, M., Srivastava, H.M., Arif, M., Ahmad, K.: Coefficient estimates for a certain family of analytic functions involving a q-derivative operator. Ramanujan J. 55, 53-71 (2021)

27. Rehman, M.S., Ahmad, Q.Z., Srivastava, H.M., Khan, B., Khan, N.: Partial sums of generalized q-Mittag-Leffler functions. AlMS Math. 5, 408-420 (2020)

28. Rehman, M.S., Ahmad, Q.Z., Srivastava, H.M., Khan, N., Darus, M., Khan, B.: Applications of higher-order q-derivatives to the subclass of $q$-starlike functions associated with the Janowski functions. AIMS Math. 6, 1110-1125 (2021)

29. Robertson, M.S.: Certain classes of starlike functions. Mich. Math. J. 32, 135-140 (1985)

30. Ruscheweyh, S.: New criteria for univalent functions. Proc. Am. Math. Soc. 49, 109-115 (1975)

31. Shi, L., Khan, Q., Srivastava, G., Liu, J.-L., Arif, M.: A study of multivalent $q$-starlike functions connected with circular domain. Mathematics 7, Article ID 670 (2019)

32. Srivastava, H.M.: Univalent functions, fractional calculus, and associated generalized hypergeometric functions. In: Srivastava, H.M., Owa, S. (eds.) Univalent Functions, Fractional Calculus, and Their Applications, pp. 329-354. Ellis Horwood, Chichester (1989)

33. Srivastava, H.M.: Operators of basic (or q-) calculus and fractional $q$-calculus and their applications in geometric function theory of complex analysis. Iran. J. Sci. Technol. Trans. A, Sci. 44, 327-344 (2020)

34. Srivastava, H.M., Ahmad, Q.Z., Khan, N., Khan, N., Khan, B.: Hankel and Toeplitz determinants for a subclass of $q$-starlike functions associated with a general conic domain. Mathematics 7, Article ID 181 (2019)

35. Srivastava, H.M., Aouf, M.K., Mostafa, A.O.: Some properties of analytic functions associated with fractional $q$-calculus operators. Miskolc Math. Notes 20, 1245-1260 (2019) 
36. Srivastava, H.M., Arif, M., Raza, M.: Convolution properties of meromorphically harmonic functions defined by a generalized convolution q-derivative operator. AIMS Math. 6, 5869-5885 (2021)

37. Srivastava, H.M., Arjika, S., Kelil, A.S.: Some homogeneous q-difference operators and the associated generalized Hahn polynomials. Appl. Set-Valued Anal. Optim. 1, 187-201 (2019)

38. Srivastava, H.M., Bansal, D.: Close-to-convexity of a certain family of q-Mittag-Leffler functions. J. Nonlinear Var. Anal. 1, 61-69 (2017)

39. Srivastava, H.M., Cao, J., Arjika, S.: A note on generalized q-difference equations and their applications involving q-hypergeometric functions. Symmetry 12, Article ID 1816 (2020)

40. Srivastava, H.M., Arjika, S.: A general family of q-hypergeometric polynomials and associated generating functions. Mathematics 9, Article ID 1161 (2021)

41. Srivastava, H.M., Karlsson, P.W.: Multiple Gaussian Hypergeometric Series. Ellis Horwood, Chichester (1985)

42. Srivastava, H.M., Khan, B., Khan, N., Ahmad, Q.Z.: Coefficient inequalities for $q$-starlike functions associated with the Janowski functions. Hokkaido Math. J. 48, 407-425 (2019)

43. Srivastava, H.M., Khan, B., Khan, N., Ahmad, Q.Z., Tahir, M.: A generalized conic domain and its applications to certain subclasses of analytic functions. Rocky Mt. J. Math. 49, 2325-2346 (2019)

44. Srivastava, H.M., Khan, B., Khan, N., Tahir, M., Ahmad, S., Khan, N.: Upper bound of the third Hankel determinant for a subclass of $q$-starlike functions associated with the $q$-exponential function. Bull. Sci. Math. 167, Article ID 102942 (2021)

45. Srivastava, H.M., Khan, S., Ahmad, Q.Z., Khan, N., Hussain, S.: The Faber polynomial expansion method and its application to the general coefficient problem for some subclasses of bi-univalent functions associated with a certain q-integral operator. Stud. Univ. Babeş-Bolyai, Math. 63, 419-436 (2018)

46. Srivastava, H.M., Raza, N., AbuJarad, E.S.A., Srivastava, G., AbuJarad, M.H.: Fekete-Szegö inequality for classes of $(p, q)$-starlike and $(p, q)$-convex functions. Rev. R. Acad. Cienc. Exactas Fís. Nat., Ser. A Mat. 113, 3563-3584 (2019)

47. Srivastava, H.M., Tahir, M., Khan, B., Ahmad, Q.Z., Khan, N.: Some general classes of $q$-starlike functions associated with the Janowski functions. Symmetry 11, Article ID 292 (2019)

48. Srivastava, H.M., Tahir, M., Khan, B., Ahmad, Q.Z., Khan, N.: Some general families of $q$-starlike functions associated with the Janowski functions. Filomat 33, 2613-2626 (2019)

49. Xu, Q.-H., Yang, T., Srivastava, H.M.: Sufficient conditions for a general class of Carathéodory functions. Filomat 30 3615-3625 (2016)

\section{Submit your manuscript to a SpringerOpen ${ }^{\circ}$ journal and benefit from:}

- Convenient online submission

- Rigorous peer review

- Open access: articles freely available online

- High visibility within the field

- Retaining the copyright to your article

Submit your next manuscript at $\boldsymbol{~ s p r i n g e r o p e n . c o m ~}$ 